\title{
Évaluation et validation de l'éducation à
} l'environnement urbain

12 - 14 septembre 1999 - Séminaire organisé par le réseau national Citéphile, Besançon (France)

Henry-George Madelaine

\section{OpenEdition}

Journals

Édition électronique

URL : http://journals.openedition.org/ere/6877

DOI : $10.4000 /$ ere.6877

ISSN : 2561-2271

Éditeur

Centr'ERE

Référence électronique

Henry-George Madelaine, «Évaluation et validation de l'éducation à l'environnement urbain », Éducation relative à l'environnement [En ligne], Volume 2| 2000, mis en ligne le 15 septembre 2000, consulté le 17 avril 2021. URL : http://journals.openedition.org/ere/6877 ; DOI : https://doi.org/ 10.4000/ere.6877 


\section{Évaluation et validation de l'éducation à l'environnement urbain}

12 - 14 septembre 1999 - Séminaire organisé par le réseau national Citéphile, Besançon (France)

Henry-George Madelaine

1 Citéphile est un réseau national français créé en 1991 à l'initiative de praticiens de l'Éducation à l'Environnement urbain pour répondre à leurs questions sur les fondements et les modalités de leurs actions. Parmi les valeurs qui sont ses références, on peut citer le croisement de regards principalement issus des champs de l'environnement, du social et de la culture, mais sans exclusive pour les autres domaines tels que, par exemple, l'économie. Une des originalités de ce réseau repose donc sur le fait que ses membres ne sauraient, par définition, être des spécialistes exclusifs de l'éducation à l'environnement urbain. Ses actions se composent de rencontres et échanges autour des pratiques, réflexions et définition de référentiels partagés autour des concepts fondateurs, formations - actions - recherches développés en coordination.

2 C'est dans cet esprit et en prolongement de travaux initiés lors du deuxième Carrefour National (Arras, Déc. 1998, actes disponibles au secrétariat général du réseau), que Citéphile a organisé un séminaire de réflexion dont le thème était: "évaluation et validation de l'éducation à l'environnement urbain». Les travaux, qui se sont rapidement concentrés sur le sujet de l'évaluation, constatant que la validation des actions n'en était qu'une résultante circonstancielle, furent introduits par un ensemble de contributions proposées par Jean-Noël Petit, chargé de mission à la DIREN (Direction Régionale de l'Environnement) du Nord - Pas de Calais, Christine Partoune, formatrice à l'Institut d'Écopédagogie de Liège et enseignante à l'Université, Claude Larivière, enseignant chercheur à l'École de travail Social de l'Université de Montréal, Francine Pellaud, enseignante chercheurs à l'Université de Genève, Federico Cunat, chercheur à FIFRE-SI et enseignant à l'École Centrale de Lille, Béatrice Auxent, architecte et chargée 
de mission au CAUE (Conseil en Architecture Urbanisme et Environnement) du Nord et Henry-George Madelaine, secrétaire général de l'agence culturelle régionale Nord - Pas de Calais.

3 Les conclusions majeures de cette rencontre peuvent être ainsi définies :

- L'évaluation est un des éléments constitutifs de l'action, ses critères ne peuvent être définis en dehors de l'action même, ils ne peuvent être généralisés ni devenir « autonomes ».

- C'est un levier pour l'action et non une sanction.

- Elle n'est pas forcément consensuelle, mais elle doit être concertée entre tous les partenaires de l'action. Ce souci doit être à la fois préalable et permanent.

- Elle doit être à la fois globale et spécialisée.

4 Les conditions de mise en oeuvre de l'action pour garantir une bonne évaluation sont, entre autres :

- une bonne estimation du contexte d'origine pour articuler l'estimation finale ;

- des critères et des objectifs clairs, définis a priori ;

- un référentiel construit ;

- une mémoire continue de l'action;

- l'association d'un regard extérieur, mais partenaire.

5 L'évaluation suppose donc:

- une opérationnalisation dans l'action elle-même ;

- la définition d'outils spécifiques ;

- une implication de tous les opérateurs, une dynamique interactive et une synergie dans les initiatives ;

- une instance permanente et un suivi constant du déroulement de l'action ;

- une visibilité et une transparence des résultats articulées sur une restitution partagée et ouverte.

Ce séminaire de réflexion va donner lieu à la publication d'annales détaillées dans le courant de l'an 2000 et a servi de cadre méthodologique à une recherche - action pilotée par des membres du réseau Citéphile autour du concept de "Classes de Villes " tel qu'il est proposé par le Ministère français à la Ville. Ce travail de réflexion, basé sur des actions expérimentales, a permis de définir un cahier des charges conforme aux critères de l'éducation à l'environnement urbain qui sera également bientôt disponible.

7 Pour tout renseignement complémentaire, veuillez-vous adresser au secrétariat général de Citéphile : Écosystèmes, 16, rue Henri Kolb, 59800 Lille, France.

\section{AUTEUR}

\section{HENRY-GEORGE MADELAINE}

Président de Citéphile 